\title{
On Decay of Solutions to Systems of Integro-differential Equations with Strongly Damped
}

\author{
Erhan Pişkin
}

Department of Mathematics, University of Dicle, Diyarbakir, Turkey

${ }^{*}$ Corresponding Author: episkin@dicle.edu.tr

Copyright (C)2013 Horizon Research Publishing All rights reserved.

\begin{abstract}
We study the system of nonlinear integro-differential equations with strong damping and weak damping terms, in a bounded domain with the initial and Dirichlet boundary conditions. The existence of global solutions by using the potential well method, and the energy decay estimate by applying a lemma of Komornik [3]
\end{abstract}

Keywords Decay, Integro-differential Equations, Strong Damping

Mathematics Subject Classification (2010): 35B40, 35R09

\section{Introduction}

In this paper we consider the following initial-boundary value problem

$$
\begin{cases}u_{t t}-\triangle u+\int_{0}^{t} g_{1}(t-\tau) \triangle u(\tau) d \tau-\triangle u_{t}-\triangle u_{t t}+u_{t}=f_{1}(u, v), & (x, t) \in \Omega \times(0, T), \\ v_{t t}-\triangle v+\int_{0}^{t} g_{2}(t-\tau) \triangle v(\tau) d \tau-\triangle v_{t}-\triangle v_{t t}+v_{t}=f_{2}(u, v), & (x, t) \in \Omega \times(0, T), \\ u(x, t)=v(x, t)=0, & x \in \partial \Omega, \\ u(x, 0)=u_{0}(x), u_{t}(x, 0)=u_{1}(x), & x \in \Omega, \\ v(x, 0)=v_{0}(x), v_{t}(x, 0)=v_{1}(x), & x \in \Omega,\end{cases}
$$

where $\Omega$ is a bounded domain with smooth boundary $\partial \Omega$ in $R^{n} ; f_{i}(.,):. R^{2} \longrightarrow R$ are given functions to be specified later.

A single wave equation of the problem (1) becomes as following

$$
u_{t t}-\triangle u+\int_{0}^{t} g_{1}(t-\tau) \triangle u(\tau) d \tau-\triangle u_{t}-\triangle u_{t t}+u_{t}=|u|^{r-1} u .
$$


The global existence and blow up of solution for (2) were established [7]. In the absence of the dispersive term $\triangle u_{t t}$ and the weak damping term $u_{t}$, were established [8]. In the absence of the dispersive term $\triangle u_{t t}$ and the strong damping term $\triangle u_{t}$, were established [11, 9]. Also, Liang and Gao [12] studied the global existence, decay and blow up of solution problem (2) with the absence of the dispersive term $\triangle u_{t t}$.

Liang and Gao [10] studied the global existence, decay and blow up of solution problem (1) with the absence of the dispersive term $\triangle u_{t t}$ and the weak damping term $u_{t}$.

In this paper, under some restrictions on the initial data, we establish the global existence and the decay of solutions.

This paper is organized as follows. In section 2, we present some lemmas, and the local existence theorem. In section 3, the global existence and energy decay of the solution are given.

\section{Preliminaries}

In this section, we shall give some assumptions and lemmas which will be used throughout this work. Let $\|$. and $\|.\|_{p}$ denote the usual $L^{2}(\Omega)$ norm and $L^{p}(\Omega)$ norm, respectively. First, we make the following assumptions:

(A1) $g_{i}(t): R^{+} \longrightarrow R^{+}$belong to $C^{1}\left(R^{+}\right)$and satisfy

$$
g_{i}(t) \geq 0, \quad g_{i}^{\prime}(t) \leq 0, \quad \text { for } t \geq 0
$$

and

$$
1-\int_{0}^{\infty} g_{i}(s) d s=l_{i}>0 .
$$

Concerning the functions $f_{1}(u, v)$ and $f_{2}(u, v)$, we take

$$
\begin{aligned}
& f_{1}(u, v)=\left[k|u+v|^{2(r+1)}(u+v)+l|u|^{r} u|v|^{r+2}\right], \\
& f_{2}(u, v)=\left[k|u+v|^{2(r+1)}(u+v)+l|u|^{r+2}|v|^{r} v\right],
\end{aligned}
$$

where $k, l>0$ are constants and $r$ satisfies

$$
\left\{\begin{array}{c}
-1<r \quad \text { if } n=1,2, \\
-1<r \leq \frac{3-n}{n-2} \quad \text { if } n \geq 3
\end{array}\right.
$$

According to the above equalities they can easily verify that

$$
u f_{1}(u, v)+v f_{2}(u, v)=2(r+2) F(u, v), \forall(u, v) \in R^{2},
$$

where

$$
F(u, v)=\frac{1}{2(r+2)}\left[k|u+v|^{2(r+2)}+2 l|u v|^{r+2}\right] .
$$

We have the following result.

Lemma 1 [5]. There exist two positive constants $c_{0}$ and $c_{1}$ such that

$$
c_{0}\left(|u|^{2(r+2)}+|v|^{2(r+2)}\right) \leq 2(r+2) F(u, v) \leq c_{1}\left(|u|^{2(r+2)}+|v|^{2(r+2)}\right)
$$

is satisfied.

Let's define

$$
\begin{aligned}
J(t)= & \frac{1}{2}\left[\left(1-\int_{0}^{t} g_{1}(\tau) d \tau\right)\|\nabla u\|^{2}+\left(1-\int_{0}^{t} g_{2}(\tau) d \tau\right)\|\nabla v\|^{2}\right] \\
& +\frac{1}{2}\left[\left(g_{1} \circ \nabla u\right)(t)+\left(g_{2} \circ \nabla v\right)(t)\right]-\int_{\Omega} F(u, v) d x
\end{aligned}
$$




$$
\begin{aligned}
I(t)= & \left(1-\int_{0}^{t} g_{1}(\tau) d \tau\right)\|\nabla u\|^{2}+\left(1-\int_{0}^{t} g_{2}(\tau) d \tau\right)\|\nabla v\|^{2} \\
& +\left[\left(g_{1} \circ \nabla u\right)(t)+\left(g_{2} \circ \nabla v\right)(t)\right]-2(r+2) \int_{\Omega} F(u, v) d x
\end{aligned}
$$

and also the energy function as follows

$$
\begin{aligned}
E(t)= & \frac{1}{2}\left(\left\|u_{t}\right\|^{2}+\left\|v_{t}\right\|^{2}\right)+\frac{1}{2}\left(\left\|\nabla u_{t}\right\|^{2}+\left\|\nabla v_{t}\right\|^{2}\right) \\
& +\frac{1}{2}\left[\left(1-\int_{0}^{t} g_{1}(\tau) d \tau\right)\|\nabla u\|^{2}+\left(1-\int_{0}^{t} g_{2}(\tau) d \tau\right)\|\nabla v\|^{2}\right] \\
& +\frac{1}{2}\left[\left(g_{1} \circ \nabla u\right)(t)+\left(g_{2} \circ \nabla v\right)(t)\right]-\int_{\Omega} F(u, v) d x,
\end{aligned}
$$

where

$$
\left(g_{i} \circ w\right)(t)=\int_{0}^{t} g_{i}(t-\tau)\|w(t)-w(\tau)\|^{2} d \tau .
$$

Lemma $2 E(t)$ is a nonincreasing function for $t \geq 0$ and

$$
\begin{aligned}
E^{\prime}(t)= & -\left(\left\|u_{t}\right\|^{2}+\left\|v_{t}\right\|^{2}\right)-\left(\left\|\nabla u_{t}\right\|^{2}+\left\|\nabla v_{t}\right\|^{2}\right) \\
& +\frac{1}{2}\left[\left(g_{1}^{\prime} \circ \nabla u\right)(t)+\left(g_{2}^{\prime} \circ \nabla v\right)\right]-\frac{1}{2}\left[g_{1}(t)\|\nabla u\|^{2}+g_{2}(t)\|\nabla v\|^{2}\right] \\
\leq & 0 .
\end{aligned}
$$

Proof. The proof is almost the same that of [5], so omit it here.

Moreover, the following energy inequality holds:

$$
E(t)+\int_{s}^{t}\left(\left\|u_{\tau}\right\|^{2}+\left\|v_{\tau}\right\|^{2}+\left\|\nabla u_{\tau}\right\|^{2}+\left\|\nabla v_{\tau}\right\|^{2}\right) d \tau \leq E(s), \text { for } 0 \leq s \leq t<T .
$$

Lemma 3 (Sobolev-Poincare inequality) [1]. Let $p$ be a number with $2 \leq p<\infty(n=1,2)$ or $2 \leq p \leq 2 n /(n-2)$ $(n \geq 3)$, then there is a constant $C_{*}=C_{*}(\Omega, p)$ such that

$$
\|u\|_{p} \leq C_{*}\|\nabla u\| \text { for } u \in H_{0}^{1}(\Omega) .
$$

The following integral inequality plays an important role in our proof of the energy decay of the solutions to problem (1).

Lemma 4 [3]. Let $h:[0, \infty) \longrightarrow[0, \infty)$ be a nonincreasing function and assume that there exists a constant $c>0$ such that

$$
\int_{t}^{\infty} h(\tau) d \tau \leq c h(t), \forall t \in[0, \infty)
$$

Then we have

$$
h(t) \leq h(0) e^{1-t c^{-1}}, \forall t \geq c
$$

Next, we state the local existence theorem that can be established by combining arguments of $[2,4]$.

Theorem 5 (Local existence). Suppose that (3) holds, and further $\left(u_{0}, v_{0}\right) \in H_{0}^{1}(\Omega) \times H_{0}^{1}(\Omega),\left(u_{1}, v_{1}\right) \in H_{0}^{1}(\Omega) \times$ $H_{0}^{1}(\Omega)$. Then problem (1) has a unique local solution

$$
u, v \in C\left([0, T) ; H_{0}^{1}(\Omega)\right) \text { and } u_{t}, v_{t} \in C\left([0, T) ; H_{0}^{1}(\Omega)\right)
$$

Moreover, at least one of the following statements holds true:

i) $T=\infty$,

ii) $\left\|u_{t}\right\|^{2}+\left\|v_{t}\right\|^{2}+l_{1}\|\nabla u\|^{2}+l_{2}\|\nabla v\|^{2} \longrightarrow \infty$ as $t \longrightarrow T^{-}$.

Remark 6 We denote by $C$ various positive constants which may be different at different occurrences. 


\section{Global existence and energy decay}

In this section, we consider the global existence and energy decay of solutions for problem (1).

Lemma 7 [5]. Suppose that (3) holds. Then there exists $\eta>0$ such that for any $(u, v) \in H_{0}^{1}(\Omega) \times H_{0}^{1}(\Omega)$, the inequality

$$
\|u+v\|_{2(r+2)}^{2(r+2)}+2\|u v\|_{r+2}^{r+2} \leq \eta\left(l_{1}\|\nabla u\|^{2}+l_{2}\|\nabla v\|^{2}\right)^{r+2}
$$

is satisfied.

For the sake of simplicity and to prove our result, we take $k=l=1$ and introduce

$$
B=\eta^{\frac{1}{2(r+2)}}, \alpha_{*}=B^{-\frac{r+2}{r+1}}, E_{1}=\left(\frac{1}{2}-\frac{1}{2(r+2)}\right) \alpha_{*}^{2},
$$

where $\eta$ is the optimal constant in (11). Next, we will state and prove a lemma which is similar to the one introduced firstly by Vitillaro in [6] to study a class of a single wave equation.

Lemma 8 Suppose that (3) holds. Let $(u, v)$ be the solution of system (1). Assume further that $E(0)<E_{1}$ and

$$
\left(l_{1}\left\|\nabla u_{0}\right\|^{2}+l_{2}\left\|\nabla v_{0}\right\|^{2}\right)^{\frac{1}{2}}<\alpha_{*}
$$

Then

$$
\left(l_{1}\|\nabla u\|^{2}+l_{2}\|\nabla v\|^{2}+\left(g_{1} \circ \nabla u\right)(t)+\left(g_{2} \circ \nabla v\right)(t)\right)^{\frac{1}{2}}<\alpha_{*},
$$

for all $t \in[0, T)$.

Proof. First from (8), (9), (11) and the definition of $B$, we have

$$
\begin{aligned}
E(t) \geq & \frac{1}{2}\left[l_{1}\|\nabla u\|^{2}+l_{2}\|\nabla v\|^{2}+\left(g_{1} \circ \nabla u\right)(t)+\left(g_{2} \circ \nabla v\right)(t)\right]-\int_{\Omega} F(u, v) d x \\
= & \frac{1}{2}\left[l_{1}\|\nabla u\|^{2}+l_{2}\|\nabla v\|^{2}+\left(g_{1} \circ \nabla u\right)(t)+\left(g_{2} \circ \nabla v\right)(t)\right] \\
& -\frac{1}{2(r+2)}\left(\|u+v\|_{2(r+2)}^{2(r+2)}+2\|u v\|_{r+2}^{r+2}\right) \\
\geq & \frac{1}{2}\left[l_{1}\|\nabla u\|^{2}+l_{2}\|\nabla v\|^{2}+\left(g_{1} \circ \nabla u\right)(t)+\left(g_{2} \circ \nabla v\right)(t)\right] \\
& -\frac{1}{2(r+2)} \eta\left(l_{1}\|\nabla u\|^{2}+l_{2}\|\nabla v\|^{2}\right)^{r+2} \\
\geq & \frac{1}{2}\left[l_{1}\|\nabla u\|^{2}+l_{2}\|\nabla v\|^{2}+\left(g_{1} \circ \nabla u\right)(t)+\left(g_{2} \circ \nabla v\right)(t)\right] \\
& -\frac{B^{2(r+2)}}{2(r+2)}\left(l_{1}\|\nabla u\|^{2}+l_{2}\|\nabla v\|^{2}\right)^{r+2} .
\end{aligned}
$$

So, we get

$$
E(t) \geq G\left(l_{1}\|\nabla u\|^{2}+l_{2}\|\nabla v\|^{2}+\left(g_{1} \circ \nabla u\right)(t)+\left(g_{2} \circ \nabla v\right)(t)\right) \text { for } t \geq 0,
$$

where $G(\alpha)=\frac{1}{2} \alpha^{2}-\frac{B^{2(r+2)}}{2(r+2)} \alpha^{2(r+2)}$. Note that $G(\alpha)$ has the maximum at $\alpha_{*}=B^{-\frac{r+2}{r+1}}$ and maximum value is

$$
E_{1}=G\left(\alpha_{*}\right)=\left(\frac{1}{2}-\frac{1}{2(r+2)}\right) \alpha_{*}^{2} .
$$

Now we establish (14) by contradiction. Suppose (14) does not hold, then it follows from the continuity of $(u(t), v(t))$ that there exists $t_{0} \in(0, T)$ such that

$$
\left(l_{1}\|\nabla u\|^{2}+l_{2}\|\nabla v\|^{2}+\left(g_{1} \circ \nabla u\right)(t)+\left(g_{2} \circ \nabla v\right)(t)\right)^{\frac{1}{2}}=\alpha_{*} .
$$

By (15), we see that

$$
E\left(t_{0}\right) \geq G\left[\left(l_{1}\left\|\nabla u\left(t_{0}\right)\right\|^{2}+l_{2}\left\|\nabla v\left(t_{0}\right)\right\|^{2}+\left(g_{1} \circ \nabla u\right)\left(t_{0}\right)+\left(g_{2} \circ \nabla v\right)\left(t_{0}\right)\right)^{\frac{1}{2}}\right]=G\left(\alpha_{*}\right)=E_{1} .
$$

This is impossible since $E(t) \leq E(0)<E_{1}, t \geq 0$. Hence (14) is established. 
Theorem 9 (Global existence and energy decay). Assume that (3) hold. If the initial data $\left(u_{0}, u_{1}\right) \in H_{0}^{1}(\Omega) \times$ $H_{0}^{1}(\Omega),\left(v_{0}, v_{1}\right) \in H_{0}^{1}(\Omega) \times H_{0}^{1}(\Omega)$, satisfy $E(0)<E_{1}$ and

$$
\left(\left\|\nabla u_{0}\right\|^{2}+\left\|\nabla v_{0}\right\|^{2}\right)^{\frac{1}{2}}<\alpha_{*}
$$

where the constants $\alpha_{*}$ and $E_{1}$ are defined in (12), then the corresponding solution to system (1) globally exists, i.e., $T=\infty$.

Moreover, if

$$
1-\eta\left(\frac{2(r+2)}{r+1} E(0)\right)^{r+1}-\frac{5(1-l)(r+2)}{2 l(r+1)}>0,
$$

then we have the following decay estimates

$$
E(t) \leq E(0) e^{1-\mu C_{7}^{-1} t}
$$

for every $t \geq \frac{C_{7}}{\mu}$, where $C_{7}$ is positive constant.

Proof. First, we prove that $T=\infty$. Since $E(0)<E_{1}$ and

$$
\left(l_{1}\left\|\nabla u_{0}\right\|^{2}+l_{2}\left\|\nabla v_{0}\right\|^{2}\right)^{\frac{1}{2}}<\alpha_{*},
$$

it follows from Lemma 8 that

$$
\begin{aligned}
& l_{1}\|\nabla u\|^{2}+l_{1}\|\nabla v\|^{2} \\
\leq & l_{1}\|\nabla u\|^{2}+l_{2}\|\nabla v\|^{2}+\left(g_{1} \circ \nabla u\right)(t)+\left(g_{2} \circ \nabla v\right)(t) \\
< & \alpha_{*}^{2}=\eta^{-\frac{1}{r+1}}
\end{aligned}
$$

which implies that

$$
\begin{aligned}
I(t) & =l_{1}\|\nabla u\|^{2}+l_{2}\|\nabla v\|^{2}+\left(g_{1} \circ \nabla u\right)(t)+\left(g_{2} \circ \nabla v\right)(t)-2(r+2) \int_{\Omega} F(u, v) d x \\
& =l_{1}\|\nabla u\|^{2}+l_{2}\|\nabla v\|^{2}-\left(\|u+v\|_{2(r+2)}^{2(r+2)}+2\|u v\|_{r+2}^{r+2}\right) \\
& \geq l_{1}\|\nabla u\|^{2}+l_{2}\|\nabla v\|^{2}-\eta\left(l_{1}\|\nabla u\|^{2}+l_{2}\|\nabla v\|^{2}\right)^{r+2} \geq 0
\end{aligned}
$$

for $t \in[0, T)$. Furthermore, (7) and (8), we get

$$
\begin{aligned}
J(t) & =\frac{r+1}{2(r+2)}\left(l_{1}\|\nabla u\|^{2}+l_{2}\|\nabla v\|^{2}+\left(g_{1} \circ \nabla u\right)(t)+\left(g_{2} \circ \nabla v\right)(t)\right)+\frac{1}{2(r+2)} I(t) \\
& \geq \frac{r+1}{2(r+2)}\left(l_{1}\|\nabla u\|^{2}+l_{2}\|\nabla v\|^{2}+\left(g_{1} \circ \nabla u\right)(t)+\left(g_{2} \circ \nabla v\right)(t)\right) .
\end{aligned}
$$

From $(10)$ and $E(t) \leq E(0)$, we deduce that

$$
\begin{aligned}
l_{1}\|\nabla u\|^{2}+l_{2}\|\nabla v\|^{2}+\left(g_{1} \circ \nabla u\right)(t)+\left(g_{2} \circ \nabla v\right)(t) & \leq \frac{2(r+2)}{r+1} J(t) \\
& \leq \frac{2(r+2)}{r+1} E(t) \\
& \leq \frac{2(r+2)}{r+1} E(0)
\end{aligned}
$$

for $t \in[0, T)$. So it follows from (23) and (10)

$$
\begin{aligned}
& \frac{r+1}{2(r+2)}\left(l_{1}\|\nabla u\|^{2}+l_{2}\|\nabla v\|^{2}+\left(g_{1} \circ \nabla u\right)(t)+\left(g_{2} \circ \nabla v\right)(t)\right)+\frac{1}{2}\left(\left\|u_{t}\right\|^{2}+\left\|v_{t}\right\|^{2}\right) \\
\leq & J(t)+\frac{1}{2}\left(\left\|u_{t}\right\|^{2}+\left\|v_{t}\right\|^{2}+\left\|\nabla u_{t}\right\|^{2}+\left\|\nabla v_{t}\right\|^{2}\right) \\
= & E(t) \leq E(0)<E_{1}, \forall t \in[0, T)
\end{aligned}
$$


which implies

$$
l_{1}\|\nabla u\|^{2}+l_{2}\|\nabla v\|^{2}+\left\|u_{t}\right\|^{2}+\left\|v_{t}\right\|^{2}<C^{\prime} E_{1}
$$

where $C^{\prime}=\max \left\{\frac{1}{2}, \frac{2(r+2)}{r+1}\right\}$. Then, by Theorem 5 , we have the global existence result.

Next, we want to derive the decay rate of energy function for problem (1). Multiplying the first equation of system (1) by $u$ and the second equation of system (1) by $v$, integrating them over $\Omega \times\left[t_{1}, t_{2}\right]\left(0 \leq t_{1} \leq t_{2}\right)$, using integration by parts and summing up, we have

$$
\begin{aligned}
& \left.\int_{\Omega} u u_{t} d x\right|_{t_{1}} ^{t_{2}}-\int_{t_{1}}^{t_{2}}\left\|u_{t}\right\|^{2} d t+\left.\int_{\Omega} v v_{t} d x\right|_{t_{1}} ^{t_{2}}-\int_{t_{1}}^{t_{2}}\left\|v_{t}\right\|^{2} d t \\
& \left.\int_{\Omega} \nabla u \nabla u_{t} d x\right|_{t_{1}} ^{t_{2}}-\int_{t_{1}}^{t_{2}}\left\|\nabla u_{t}\right\|^{2} d t+\left.\int_{\Omega} \nabla v \nabla v_{t} d x\right|_{t_{1}} ^{t_{2}}-\int_{t_{1}}^{t_{2}}\left\|\nabla v_{t}\right\|^{2} d t \\
& +\int_{t_{1}}^{t_{2}}\|\nabla u\|^{2} d t+\int_{t_{1}}^{t_{2}}\|\nabla v\|^{2} d t \\
& +\int_{t_{1}}^{t_{2}} \int_{\Omega}^{t_{2}} u u_{t} d x d t+\int_{t_{1}}^{t_{2}} \int_{\Omega} v v_{t} d x d t+\int_{t_{1}}^{t_{2}} \int_{\Omega} \nabla u \nabla u_{t} d x d t+\int_{t_{1}}^{t_{2}} \int_{\Omega} \nabla v \nabla v_{t} d x d t \\
& +\int_{0}^{t} \int_{t_{1}}^{t_{2}} \int_{\Omega} g_{1}(t-\tau) \triangle u(\tau) d \tau u(t) d x d t+\int_{0}^{t_{2}} \int_{t_{1}} g_{\Omega}(t-\tau) \triangle v(\tau) d \tau v(t) d x d t \\
& =\int_{t_{1}}^{t_{2}}\left[u f_{1}(u, v)+v f_{2}(u, v)\right] d x d t .
\end{aligned}
$$

It follows from $(9)$

$$
\begin{aligned}
& 2 \int_{t_{1}}^{t_{2}} E(t) d t-2(r+1) \int_{t_{1}}^{t_{2}} \int_{\Omega} F(u, v) d x d t \\
= & -\left.\int_{\Omega}\left(u u_{t}+v v_{t}\right) d x\right|_{t_{1}} ^{t_{2}}+2 \int_{t_{1}}^{t_{2}}\left(\left\|u_{t}\right\|^{2}+\left\|v_{t}\right\|^{2}\right) d t \\
& -\left.\int_{\Omega}^{t_{2}}\left(\nabla u \nabla u_{t}+\nabla v \nabla v_{t}\right) d x\right|_{t_{1}} ^{t_{2}}+2 \int_{t_{1}}^{t_{2}}\left(\left\|\nabla u_{t}\right\|^{2}+\left\|\nabla v_{t}\right\|^{2}\right) d t \\
& -\int_{t_{1}}^{t_{2}} \int_{\Omega}^{t_{2}}\left(u u_{t}+v v_{t}\right) d x d t+\int_{t_{1}}^{t_{2}}\left(\nabla u \nabla u_{t}+\nabla v \nabla v_{t}\right) d x d t \\
& +\int_{t_{1}}^{t_{2}}\left(g_{1} \circ \nabla u\right)(t) d t+\int_{t_{1}}^{t_{2}}\left(g_{2} \circ \nabla v\right)(t) d t \\
& -\int_{t_{1}}^{t_{2}} \int_{0}^{t} g_{1}(\tau) d \tau\|\nabla u(t)\| d t-\int_{t_{1}}^{t_{2}} \int_{0}^{t} g_{2}(\tau) d \tau\|\nabla v(t)\| d t \\
& -\int_{0}^{t} \int_{t_{1}}^{t_{2}} \int_{\Omega} g_{1}(t-\tau) \triangle u(\tau) d \tau u(t) d x d t \\
& -\int_{0}^{t} \int_{t_{1}}^{t_{2}} g_{\Omega} g_{2}(t-\tau) \triangle v(\tau) d \tau v(t) d x d t .
\end{aligned}
$$

For the eleventh term on the right hand side of $(25)$, we have

$$
\begin{aligned}
& -\int_{\Omega} \int_{0}^{t} g_{1}(t-\tau) \triangle u(\tau) u(t) d \tau d x \\
= & \int_{\Omega} \int_{0}^{t} g_{1}(t-\tau) \nabla u(\tau) \nabla u(t) d \tau d x \\
= & \frac{1}{2}\left[\int_{0}^{t} g_{1}(t-\tau)\left(\|\nabla u(t)\|^{2}+\|\nabla u(\tau)\|^{2}\right) d \tau-\left(g_{1} \circ \nabla u\right)(t)\right],
\end{aligned}
$$

and similarly, we have

$$
\begin{aligned}
& -\int_{\Omega} \int_{0}^{t} g_{2}(t-\tau) \Delta v(\tau) v(t) d \tau d x \\
= & \int_{\Omega} \int_{0}^{t} g_{2}(t-\tau) \nabla v(\tau) \nabla v(t) d \tau d x \\
= & \frac{1}{2}\left[\int_{0}^{t} g_{2}(t-\tau)\left(\|\nabla v(t)\|^{2}+\|\nabla v(\tau)\|^{2}\right) d \tau-\left(g_{2} \circ \nabla v\right)(t)\right] .
\end{aligned}
$$


Substituting (26), (27) into (25), we have

$$
\begin{aligned}
& 2 \int_{t_{1}}^{t_{2}} E(t) d t-2(r+1) \int_{t_{1}}^{t_{2}} \int_{\Omega} F(u, v) d x d t \\
& =-\left.\int_{\Omega}\left(u u_{t}+v v_{t}\right) d x\right|_{t_{1}} ^{t_{2}}+2 \int_{t_{1}}^{t_{2}}\left(\left\|u_{t}\right\|^{2}+\left\|v_{t}\right\|^{2}\right) d t \\
& -\left.\int_{\Omega}\left(\nabla u \nabla u_{t}+\nabla v \nabla v_{t}\right) d x\right|_{t_{1}} ^{t_{2}}+2 \int_{t_{1}}^{t_{2}}\left(\left\|\nabla u_{t}\right\|^{2}+\left\|\nabla v_{t}\right\|^{2}\right) d t \\
& -\int_{t_{1}}^{t_{2}} \int_{\Omega}\left(u u_{t}+v v_{t}\right) d x d t+\int_{t_{1}}^{t_{2}} \int_{\Omega}\left(\nabla u \nabla u_{t}+\nabla v \nabla v_{t}\right) d x d t \\
& +\frac{1}{2} \int_{0}^{t} g_{1}(t-\tau)\|\nabla u(\tau)\|^{2} d \tau d t+\frac{1}{2} \int_{0}^{t} g_{2}(t-\tau)\|\nabla v(\tau)\|^{2} d \tau d t \\
& +\frac{1}{2} \int_{t_{1}}^{t_{2}}\left[\left(g_{1} \circ \nabla u\right)(t)+\left(g_{2} \circ \nabla v\right)(t)\right] d t \\
& -\frac{1}{2} \int_{t_{1}}^{t_{2}}\left[\int_{0}^{t} g_{1}(\tau) d \tau\|\nabla u(t)\|^{2}+\int_{0}^{t} g_{2}(\tau) d \tau\|\nabla v(t)\|^{2}\right] d t \\
& \leq-\left.\int_{\Omega}\left(u u_{t}+v v_{t}\right) d x\right|_{t_{1}} ^{t_{2}}+2 \int_{t_{1}}^{t_{2}}\left(\left\|u_{t}\right\|^{2}+\left\|v_{t}\right\|^{2}\right) d t \\
& -\left.\int_{\Omega}\left(\nabla u \nabla u_{t}+\nabla v \nabla v_{t}\right) d x\right|_{t_{1}} ^{t_{2}}+2 \int_{t_{1}}^{t_{2}}\left(\left\|\nabla u_{t}\right\|^{2}+\left\|\nabla v_{t}\right\|^{2}\right) d t \\
& -\int_{t_{1}}^{t_{2}} \int_{\Omega}\left(u u_{t}+v v_{t}\right) d x d t+\int_{t_{1}}^{t_{2}} \int_{\Omega}\left(\nabla u \nabla u_{t}+\nabla v \nabla v_{t}\right) d x d t \\
& +\frac{1}{2} \int_{t_{1}}^{t_{2}}\left[\int_{0}^{t} g_{1}(t-\tau)\|\nabla u(\tau)\|^{2} d \tau+\int_{0}^{t} g_{2}(t-\tau)\|\nabla v(\tau)\|^{2} d \tau\right] d t \\
& +\frac{1}{2} \int_{t_{1}}^{t_{2}}\left[\left(g_{1} \circ \nabla u\right)(t)+\left(g_{2} \circ \nabla v\right)(t)\right] d t \\
& =A_{1}+A_{2}+A_{3}+A_{4}+A_{5}+A_{6}+A_{7}+A_{8} \text {. }
\end{aligned}
$$

In what follows we will estimate $A_{1}, A_{2}, \ldots, A_{8}$ in (28). Firstly, by Hölder, Young and Sobolev Poincare inequalities, we have

$$
\begin{aligned}
\int_{\Omega}\left(\left|u u_{t}\right|+\left|v v_{t}\right|\right) d x & \leq \frac{1}{2}\|u(t)\|^{2}+\frac{1}{2}\left\|u_{t}(t)\right\|^{2}+\frac{1}{2}\|v(t)\|^{2}+\frac{1}{2}\left\|v_{t}(t)\right\|^{2} \\
& \leq \frac{C}{2}\|\nabla u(t)\|^{2}+\frac{1}{2}\left\|u_{t}(t)\right\|^{2}+\frac{C}{2}\|\nabla v(t)\|^{2}+\frac{1}{2}\left\|v_{t}(t)\right\|^{2}
\end{aligned}
$$

Then, by (24), we have

$$
A_{1} \leq\left.\int_{\Omega}\left(\left|u u_{t}\right|+\left|v v_{t}\right|\right) d x\right|_{t_{1}} ^{t_{2}} \leq 2 C_{1} E\left(t_{1}\right)
$$

For $A_{2}$ in (28), applying $\left\|u_{t}\right\|^{2}+\left\|v_{t}\right\|^{2} \leq-E^{\prime}(t)$ from (10), we have

$$
A_{2}=2 \int_{t_{1}}^{t_{2}}\left(\left\|u_{t}\right\|^{2}+\left\|v_{t}\right\|^{2}\right) d t \leq 2 C_{2} E\left(t_{1}\right) .
$$

Similarly, we have

$$
A_{3} \leq\left.\int_{\Omega}\left(\left|\nabla u \nabla u_{t}\right|+\left|\nabla v \nabla v_{t}\right|\right) d x\right|_{t_{1}} ^{t_{2}} \leq 2 C_{3} E\left(t_{1}\right)
$$

and

$$
A_{4}=2 \int_{t_{1}}^{t_{2}}\left(\left\|\nabla u_{t}\right\|^{2}+\left\|\nabla v_{t}\right\|^{2}\right) d t \leq 2 C_{4} E\left(t_{1}\right) .
$$


We also have the following estimate

$$
\begin{aligned}
A_{5} & =\int_{t_{1}}^{t_{2}} \int_{\Omega}\left(u u_{t}+v v_{t}\right) d x d t \\
& =\frac{1}{2} \int_{t_{1}}^{t_{2}} \frac{d}{d t}\|u\|^{2} d t+\frac{1}{2} \int_{t_{1}}^{t_{2}} \frac{d}{d t}\|v\|^{2} d t \\
& =\frac{1}{2}\left(\left\|u\left(t_{2}\right)\right\|^{2}-\left\|u\left(t_{1}\right)\right\|^{2}\right)+\frac{1}{2}\left(\left\|v\left(t_{2}\right)\right\|^{2}-\left\|v\left(t_{1}\right)\right\|^{2}\right) \\
& \leq \frac{2(r+2)}{r+1} E\left(t_{1}\right)=C_{5} E\left(t_{1}\right) .
\end{aligned}
$$

Similarly

$$
A_{6}=\int_{t_{1}}^{t_{2}} \int_{\Omega}\left(\nabla u \nabla u_{t}+\nabla v \nabla v_{t}\right) d x d t \leq C_{6} E\left(t_{1}\right) .
$$

Using Young inequality for convolution $\|f * g\|_{1} \leq\|f\|_{1}\|g\|_{1}$, we have

$$
\begin{aligned}
A_{7} & =\frac{1}{2} \int_{t_{1}}^{t_{2}}\left[\int_{0}^{t} g_{1}(t-\tau)\|\nabla u(\tau)\|^{2} d \tau+\int_{0}^{t} g_{2}(t-\tau)\|\nabla v(\tau)\|^{2} d \tau\right] d t \\
& \leq \frac{1}{2} \int_{t_{1}}^{t_{2}}\left[\left\|g_{1} *\right\| \nabla u\left\|^{2}\right\|_{1}+\left\|g_{2} *\right\| \nabla v\left\|^{2}\right\|_{1}\right] \\
& =\frac{1}{2} \int_{t_{1}}^{t_{2}} g_{1}(t) d t \int_{t_{1}}^{t_{2}}\|\nabla u(t)\|^{2} d t+\frac{1}{2} \int_{t_{1}}^{t_{2}} g_{2}(t) d t \int_{t_{1}}^{t_{2}}\|\nabla v(t)\|^{2} d t \\
& \leq \frac{1}{2}\left(1-l_{1}\right) \int_{t_{1}}^{t_{2}}\|\nabla u(t)\|^{2} d t+\frac{1}{2}\left(1-l_{2}\right) \int_{t_{1}}^{t_{2}}\|\nabla v(t)\|^{2} d t \\
& \leq \frac{1-l}{2} \int_{t_{1}}^{t_{2}}\left(\|\nabla u(t)\|^{2}+\|\nabla v(t)\|^{2}\right) d t \\
& \leq \frac{(1-l)(r+2)}{l(r+1)} \int_{t_{1}}^{t_{2}} E(t) d t .
\end{aligned}
$$

From (23), we have

$$
\begin{aligned}
& \int_{t_{1}}^{t_{2}}\left[\int_{0}^{t} g_{1}(t-\tau)\|\nabla u(t)\|^{2} d \tau+\int_{0}^{t} g_{2}(t-\tau)\|\nabla v(t)\|^{2} d \tau\right] d t \\
\leq & \left(1-l_{1}\right) \int_{t_{1}}^{t_{2}}\|\nabla u(t)\|^{2} d t+\left(1-l_{2}\right) \int_{t_{1}}^{t_{2}}\|\nabla v(t)\|^{2} d t \\
\leq & (1-l) \int_{t_{1}}^{t_{2}}\left(\|\nabla u(t)\|^{2}+\|\nabla v(t)\|^{2}\right) d t \\
\leq & \frac{2(1-l)(r+2)}{l(r+1)} \int_{t_{1}}^{t_{2}} E(t) d t .
\end{aligned}
$$

Combining (35) and (36), we have

$$
\begin{aligned}
A_{8}= & \frac{1}{2} \int_{t_{1}}^{t_{2}}\left[\left(g_{1} \circ \nabla u\right)(t)+\left(g_{2} \circ \nabla v\right)(t)\right] d t \\
\leq & \frac{1}{2} \int_{t_{1}}^{t_{2}} \int_{0}^{t} g_{1}(t-\tau)\left(\|\nabla u(t)\|^{2}+\|\nabla u(\tau)\|^{2}\right) d \tau d t \\
& +\frac{1}{2} \int_{t_{1}}^{t_{2}} \int_{0}^{t} g_{2}(t-\tau)\left(\|\nabla v(t)\|^{2}+\|\nabla v(\tau)\|^{2}\right) d \tau d t \\
\leq & \frac{4(1-l)(r+2)}{l(r+1)} \int_{t_{1}}^{t_{2}} E(t) d t .
\end{aligned}
$$

Inserting estimates (29)-(37) into (28), we arrive at

$$
2 \int_{t_{1}}^{t_{2}} E(t) d t-2(r+1) \int_{t_{1}}^{t_{2}} \int_{\Omega} F(u, v) d x d t \leq C_{7} E\left(t_{1}\right)+\frac{5(1-l)(r+2)}{l(r+1)} \int_{t_{1}}^{t_{2}} E(t) d t .
$$

where $C_{7}=2 C_{1}+2 C_{2}+2 C_{3}+2 C_{4}+C_{5}+C_{6}$. 
On the other hand, from (11) and (23), we have

$$
\begin{aligned}
2(r+1) \int_{\Omega} F(u, v) d x & =\frac{r+1}{r+2}\left(\|u+v\|_{2(r+2)}^{2(r+2)}+2\|u v\|_{r+2}^{r+2}\right) \\
& \leq \frac{r+1}{r+2} \eta\left(l_{1}\|\nabla u\|^{2}+l_{2}\|\nabla v\|^{2}\right)^{r+2} \\
& \leq 2 \eta\left(\frac{2(r+2)}{r+1} E(0)\right)^{r+1} E(t)
\end{aligned}
$$

which implies

$$
2 \int_{t_{1}}^{t_{2}} E(t) d t-2(r+1) \int_{t_{1}}^{t_{2}} \int_{\Omega} F(u, v) d x d t \geq 2\left(1-\eta\left(\frac{2(r+2)}{r+1} E(0)\right)^{r+1}\right) \int_{t_{1}}^{t_{2}} E(t) d t .
$$

Noting that $E(0)<E_{1}$, we see

$$
1-\eta\left(\frac{2(r+2)}{r+1} E(0)\right)^{r+1}>0
$$

Thus, combining (38) and (39), we have

$$
2\left(1-\eta\left(\frac{2(r+2)}{r+1} E(0)\right)^{r+1}\right) \int_{t_{1}}^{t_{2}} E(t) d t \leq C_{7} E\left(t_{1}\right)+\frac{5(1-l)(r+2)}{l(r+1)} \int_{t_{1}}^{t_{2}} E(t) d t
$$

that is

$$
\begin{gathered}
2\left(1-\eta\left(\frac{2(r+2)}{r+1} E(0)\right)^{r+1}-\frac{5(1-l)(r+2)}{2 l(r+1)}\right) \int_{t_{1}}^{t_{2}} E(t) d t \leq C_{7} E\left(t_{1}\right) \\
\Longrightarrow \mu \int_{t_{1}}^{t_{2}} E(t) d t \leq C_{7} E\left(t_{1}\right)
\end{gathered}
$$

where $\mu=2\left(1-\eta\left(\frac{2(r+2)}{r+1} E(0)\right)^{r+1}-\frac{5(1-l)(r+2)}{2 l(r+1)}\right)$.

We rewrite (40) as

$$
\mu \int_{t}^{\infty} E(t) d t \leq C_{7} E(t)
$$

for every $t \in[0, \infty)$.

Since $\mu>0$ from the assumption of conditions, by Lemma 4 , we have

$$
E(t) \leq E(0) e^{1-\mu C_{7}^{-1} t}
$$

for every $t \geq \frac{C_{7}}{\mu}$. The proof is completed.

\section{REFERENCES}

[1] R. A. Adams, J. J. F. Fournier, Sobolev Spaces, Academic Press, 2003.

[2] V. Georgiev, G. Todorova, Existence of a solution of the wave equation with nonlinear damping and source terms, J. Differential Equations, 109(2): (1994) 295-308.

[3] V. Komornik, Exact controllability and stabilization, RAM: Research in Applied Mathematics, Masson, Paris, 1994.

[4] M.M. Cavalcanti, V.N.D. Cavalcanti, and J. Ferreira, Existence and uniform decay for nonlinear viscoelastic equation with strong damping, Math. Methods Appl. Sci. 24 (2001), pp. 1043-1053.

[5] S. A. Messaoudi, B. Said-Houari, Global nonexistence of positive initial-energy solutions of a system of nonlinear viscoelastic wave equations with damping and source terms, J. Math. Anal. Appl. 365 (2010), $277-287$. 
[6] E. Vitillaro, Global nonexistence theorems for a class of evolution equations with dissipation, Arch. Ration. Mech. Anal. 149(2): (1999) 155-182.

[7] X. Runzhang, Y. Yanbing, L. Yacheng, Global well-posedness for strongly damped viscoelastic wave equation, Appl. Anal., 92(1): (2013) 138-157.

[8] H.T. Song, C. K. Zhong,: Blow-up of solutions of a nonlinear viscoelastic wave equation. Nonlinear Ana., 11: (2010) 3877-3883.

[9] M. Kafini and S.A. Messaoudi, A blow-up result in a Cauchy viscoelastic problem, Appl. Math. Lett. 21: (2008) 549-553.

[10] F. Liang, H. Gao, Exponential energy decay and blow-up of solutions for a system of nonlinear viscoelastic wave equations with strong damping, Boundary Value Problem, 2011: (2011) 1-19.

[11] Y. Wang, Y. Wang, Exponential decay of solutions of viscoelastic wave equations, J. Math. Anal. Appl., 347: (2008) 18-25.

[12] F. Liang, H. Gao, Global existence and blow up of solutions for a nonlinear wave equation with memory, J. Ineq. Appl., 2012: (2012) 1-27. 